\title{
Total Arterial Coronary Artery Bypass Grafting - a review article with short experience in Apollo Hospitals Dhaka
}

\author{
M Quamrul Islam Talukder ${ }^{1}$
}

\begin{abstract}
Coronary Artery Bypass Surgery (CABG) is an established surgical treatment of coronary artery disease. Conventionally it has been done with left internal mammary artery and saphenous vein for many years. But the study shows that the rate of stenosis of venous graft is much higher than arterial graft in 10 years after CABG. ${ }^{1}$ Recent studies also proved that the outcome of total arterial grafts for CABG is much better than conventional CABG. ${ }^{2}$ In my recent short practice in this hospital, it also proved to be a safe procedure with better early post operative results.
\end{abstract}

\section{Materials and Methods}

I reviewed current literatures and found five of them relevant to the context. In addition we are following four cases that we have done here in the last 3 months for post operative in-hospital course and for quality of life after discharge from hospital.

\section{Introduction}

Coronary artery by pass grafting (CABG) is an established successful surgical procedure for coronary artery disease and being practiced for decades. In Bangladesh also CABG has been practiced for more than 10 years and gained patients' confidence. In short, CABG is done using alternate blood vessels, either vein or artery from patients' own body to reestablish heart's own blood supply bypassing the block in the coronary artery, so the name of the surgery is bypass surgery. For the venous graft, we use Saphenous vein (SV) from leg and for arterial graft, we use two arteries from the chest wall named internal mammary artery (IMA) and radial artery (RA) from hand. Conventionally
CABG used to be done with saphenous vein and left internal mammary artery for a long time. But with the result of early occlusion of vein grafts, total arterial bypass grafting meaning using all arterial conduits for CABG has been practiced with better outcome for the last decade.

\section{Discussion}

Saphenous vein and left internal mammary artery (LIMA) were being used for CABG as a standard practice since long (Fig1). But studies showed that, 10 years after CABG, percentage of occlusion of vein grafts are significantly higher than arterial grafts. According to the study Goldman et.al. JACC 44.11,2004, the patency of vein grafts, 10 years after CABG was found to be $60 \%$ whereas the patency of LIMA remained more than $90 \% .{ }^{1}$ In other words, in long term patients in whom vein grafts were used re-develop symptoms of coronary artery disease including heart attack requiring risky re-interventions, in significantly higher percentage than in whom arterial grafts were used.

To mitigate this disadvantage, cardiac surgeons started using all arterial grafts for total arterial coronary artery bypass grafting for the last decade and got success. For this, as a standard practice they use right and left internal mammary arteries (IMA) from the chest wall and radial artery (RA) from fore arm in different combination according to the number and position of the blocks (Fig. 2,3 and 4). As for other arterial conduit, previously right gastroepiploic artery was used by some surgeon. But the study shows that the occlusion rate of right gastroepiploic artery is similar as in a vein. ${ }^{2}$ So it is no longer recommended for CABG now a days. 


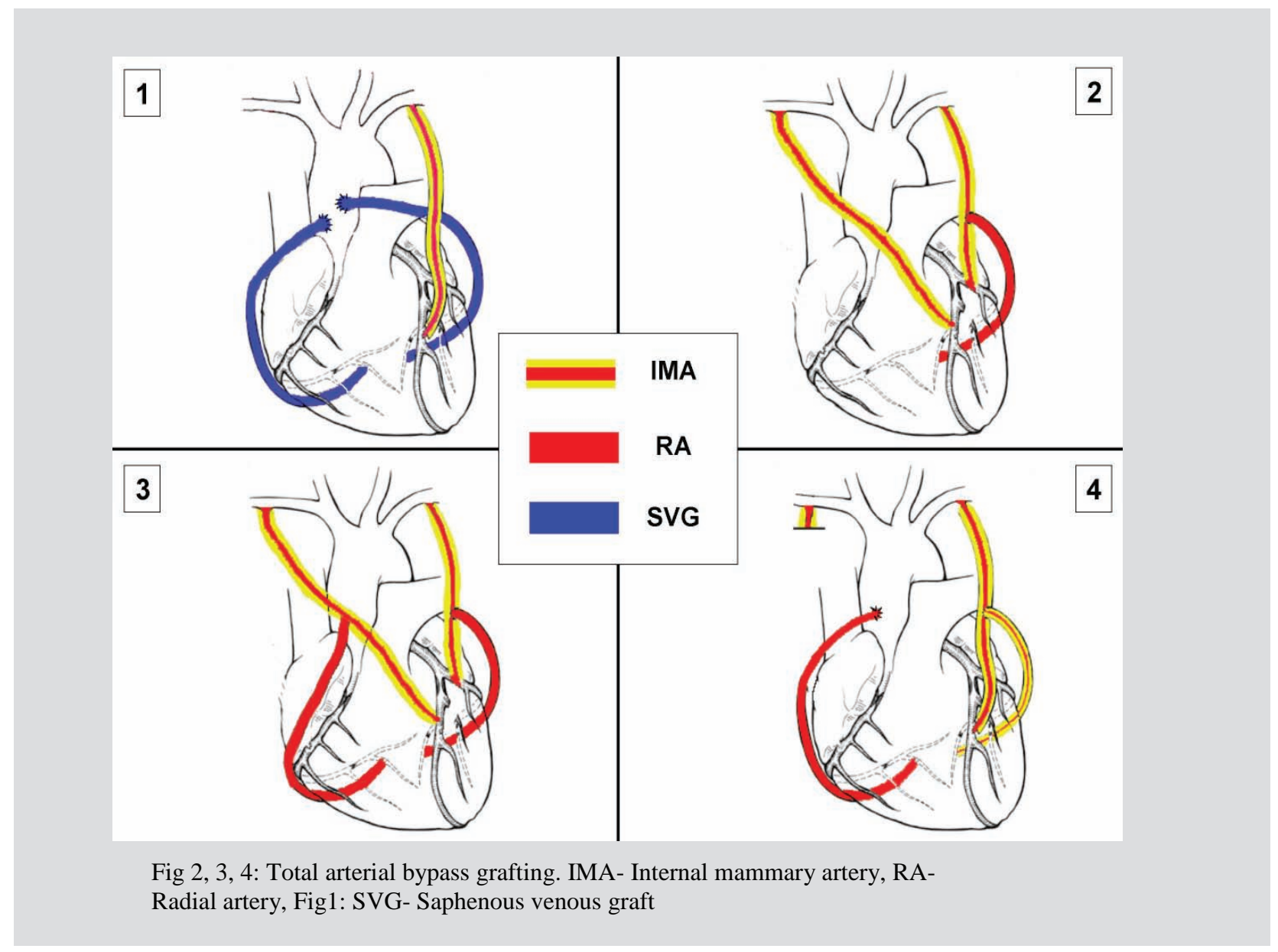

Dr. Jean - Francoise and his colleagues showed in their series that the survival and symptom free quality of life is almost double in patients with total arterial grafts than those who had conventional CABG. ${ }^{3}$ In the recent study of Malvindi et.al. ICTVS 2007, it is found that the patency of IMA and RA grafts in patients 10 years after total arterial grafts were more than $90 \%{ }^{2}$ There was a fear for taking down bilateral internal mammary for grafting for increased incidence of sternal wound complication in diabetic patients. But the study revealed that in diabetic patients also bilateral internal mammary artery can be safely used if they are skeletonized. ${ }^{4}$

Total arterial bypass grafting also has short term advantages over venous grafts. Dr. A.G. Royse showed in his study (AG Royse et. al.
EJCTS.1999;16:499-505) that post operative in-hospital mortality in case of CABG with vein grafts was $2.9 \%$ where as that was $0.2-0.9 \%$ in case of total arterial bypass grafting. ${ }^{5}$ Dr. Sundt, a scholar cardiac surgeon from the renowned hospital, Mayo Clinic, USA (I had the opportunity to work with him as an associate for long 3 years) nearly completing his study with 10 years follow up of 2000 cases with total arterial bypass grafting showing the patency of the arterial grafts remaining more than $95 \%$ at 8 years after the surgery. In the opinion of the world class scholarly cardiac surgeons like him at present, the patients with coronary artery disease with the age group around 50 or below should have total arterial bypass grafting for its long term benefit. In Bangladesh, a good percentage of patients with coronary artery 
disease fall in this group and likewise should get the benefit out of this surgery with total arterial grafting. Also because, most people here are poor and can not afford to undergo a second intervention which is sometimes risky even.

In the last three months I did 4 such CABG surgeries here with all arterial conduits. All off the patients were male within the age group of 45-52 years. All the cases were done with on-pump CABG. Average number of grafts was 3.3. All of them received bilateral skeletanized internal mammary artery and radial artery including in situ bilateral internal mammary artery in a single patient. Other three received divided right internal mammary artery anastomosed with in situ left internal mammary artery as composite grafts. All of the patients recovered well uneventfully in stipulated post operative time with preserved left ventricular ejection fraction. In the last two months follow up period they are doing extremely well in terms of symptom free good quality of life. I am hopeful to include more of such patients in my series and show the positive results on long term follow up.

\section{Conclusion}

Total arterial coronary artery bypass grafting is an established safe procedure for coronary artery disease especially for the patients with younger age group. In Bangladesh the onset of coronary artery disease occurs in early age group in comparison to western countries. I strongly believe that total arterial bypass grafting will revolutionize in the field of CABG in Bangladesh especially in the young age group in terms of long term benefits.

\section{Reference:}

1. Goldman S, Zandia K, Mortiz T, Ovitt T, Settig. Long term patency of saphanous vein and left internal mammary artery grafts after coronary artery bypass surgery. J Am Coll Cardiol. 2004;44(11):2149-56.

2. Malvindi PG, Jacob S, Kallikourdis A, Vitale N. What is the patency of gastroepiploic artery when used for coronary artery bypass grafting? Interact Cardiovasc Thorac Surg. 2007;2:397-402.

3. Jean-Francois L, Ansar H, Karen J B and John A H. The effect of total arterial grafting on medium term outcomes following coronary artery bypass grafting. Journal of Cardiothoracic Surgery. 2007;2:44.

4. Hitoshi H, Atsushi A, Syuichirou T and Akihito T. Skeletonized bilateral internal mammary artery grafting for patients with diabetes. Interact Cardiovasc Thorac Surg. 2003;2:287-292.

5. Royse AG, Royse CF, Tatoulis J. Total arterial coronary revascularization and factors influencing in hospital mortality. Eu. J. Cardiothorac Surgery. 1999;16(5):499-505. 